\title{
Improved Visibility of Metastatic Disease in the Liver During Intra-Arterial Therapy Using Delayed Arterial Phase Cone-Beam CT
}

\author{
Ruediger E. Schernthaner ${ }^{1,2} \cdot$ Reham R. Haroun ${ }^{2} \cdot$ Rafael Duran $^{2} \cdot$ \\ Howard $\mathrm{Lee}^{2}$ - Sonia Sahu' ${ }^{2}$ Jae Ho Sohn' ${ }^{2}$ Julius Chapiro ${ }^{2}$ Yan Zhao' ${ }^{2}$. \\ Boris Gorodetski ${ }^{2}$ - Florian Fleckenstein ${ }^{2} \cdot$ Susanne Smolka $^{2} \cdot$ Alessandro Radaelli ${ }^{3}$. \\ Imramsjah Martijn van der $\mathrm{Bom}^{3} \cdot{\mathrm{MingDe} \mathrm{Lin}^{2,4} \cdot \text { Jean Francois Geschwind }}^{2}$
}

Received: 13 September 2015/ Accepted: 31 May 2016/Published online: 5 July 2016

(c) The Author(s) 2016. This article is published with open access at Springerlink.com

\begin{abstract}
Purpose To compare the visibility of liver metastases on dual-phase cone-beam CT (DP-CBCT) and digital subtraction angiography (DSA), with reference to preinterventional contrast-enhanced magnetic resonance imaging (CE-MRI) of the liver.

Methods This IRB-approved, retrospective study included 28 patients with neuroendocrine (NELM), colorectal (CRCLM), or sarcoma (SLM) liver metastases who underwent DP-CBCT during intra-arterial therapy (IAT) between 01/2010 and 10/2014. DP-CBCT was acquired
\end{abstract}

Jean Francois Geschwind

jeff.geschwind@yale.edu

Ruediger E. Schernthaner

ruediger.schernthaner@meduniwien.ac.at

Reham R. Haroun

rehamharoun1989@gmail.com

Rafael Duran

rafaelduran.md@gmail.com

Howard Lee

mail2howielee@gmail.com

Sonia Sahu

sonia.p.sahu@gmail.com

Jae Ho Sohn

sohn87@gmail.com

Julius Chapiro

j.chapiro@googlemail.com

Yan Zhao

yanzhao211@163.com

Boris Gorodetski

boris.gorodetski@charite.de

Florian Fleckenstein

florian.fleckenstein@charite.de after a single contrast agent injection in the tumor-feeding arteries at early and delayed arterial phases (EAP and DAP). The visibility of each lesion was graded by two radiologists in consensus on a three-rank scale (complete, partial, none) on DP-CBCT and DSA images using CEMRI as reference.

Results 47 NELM, 43 CRCLM, and 16 SLM were included. On DSA 85.1, 44.1, and 37.5\% of NELM, CRCLM, and SLM, were at least partially depicted, respectively. EAP-CBCT yielded significantly higher sensitivities of 88.3 and $87.5 \%$ for CRCLM and SLM, respectively

Susanne Smolka

susanne.smolka@charite.de

Alessandro Radaelli

Alessandro.Radaelli@philips.com

Imramsjah Martijn van der Bom

martijn.van.der.bom@philips.com

MingDe Lin

ming.lin@philips.com

1 Section of Cardiovascular and Interventional Radiology, Department of Biomedical Imaging and Image-guided Therapy, Medical University of Vienna, Währinger Gürtel 18-20, 1090 Vienna, Austria

2 Department of Radiology and Biomedical Imaging, Yale University School of Medicine, 330 Cedar Street, TE 2-230, New Haven, CT 06520, USA

3 Image-Guided Therapy Systems, Philips Healthcare, Best, The Netherlands

4 U/S Imaging and Interventions, Philips Research North America, Cambridge, MA, USA 
$(p<0.01)$, but not for NELM $(89.4 \% ; p=1.0)$. On DAPCBCT all NELM, CRCLM, and SLM were visible $(p<0.001)$. Complete depiction was achieved on DSA for 59.6, 16.3, and $18.8 \%$ of NELM, CRCLM, and SLM, respectively. The complete depiction rate on EAP-CBCT was significantly higher for CRCLM (46.5\%; $p<0.001)$, lower for NELM (40.4\%; $p=0.592)$, and similar for SLM $(25 \%, p=0.399)$. On DAP-CBCT however, the highest rates of complete depiction were found-NELM (97.8\%; $p=0.008)$, CRCLM (95.3\%; $p=0.008)$, and SLM $(100 \% ; p<0.001)$.

Conclusion DAP-CBCT substantially improved the visibility of liver metastases during IAT. Future studies need to evaluate the clinical impact.

Keywords Interventional oncology · Transarterial chemoembolization/embolisation (TACE) · Radioembolization/radio-embolisation · Liver/hepatic . Cancer $\cdot$ Imaging

\section{Introduction}

Cancer is a major health problem, nowadays being the most common cause of death of patients younger than 85 years in developed countries [1]. Metastatic liver disease is the most common cause of malignant liver lesions [2]. Independent of the primary tumor, many patients with metastatic liver disease are not eligible for liver resection [3, 4]. In addition, many of these patients present with chemoresistant disease such that the lesions show progression despite systemic chemotherapy. And in many of these patients, the oncologic disease is liverdominant, where liver failure due to destruction of healthy liver tissue by the liver metastases is the primary limit to the patients' life-expectancy. For these patients, intraarterial therapies (IAT) such as transarterial chemoembolization (TACE) or radio-embolization (RE) are effective salvage therapies for inoperable liver metastases of different origin, such as colorectal cancer [5, 6], gastroenteropancreatic neuroendocrine tumors [7, 8], and sarcomas [9].

Contrast-enhanced magnetic resonance imaging (CE$\mathrm{MRI}$ ) is the imaging modality of choice for the diagnosis and therapy response assessment of primary and secondary liver cancer [10, 11]. Some liver metastases are hypervascular and show strong enhancement on CE-MRI, e.g., neuroendocrine liver metastases (NELM). However, most liver metastases present with a hypovascular, necrotic core and a viable, hypervascular rim, e.g., colorectal liver metastases (CRCLM). These hypovascular liver lesions are often occult or difficult to identify on digital subtraction angiography (DSA) images [12, 13], making the transition of preprocedure CE-MRI findings into the IAT often challenging, which might result in a less selective/precise IAT (e.g., lobar injection) with a higher chance of nontarget embolization and inadequate treatment.

Since the introduction of C-arm cone-beam computed tomography (CBCT) in Interventional Radiology [14, 15], this imaging modality has shown great value in the management of hepatocellular carcinoma (HCC) [16-20]. In particular, CBCT facilitates treatment planning and treatment delivery by providing a three-dimensional visualization of the tumor-feeding arteries and the capability to detect HCC lesions that are occult on DSA $[12,13]$. IAT was initially developed for the treatment of HCC, so that most procedures are nowadays performed in patients with HCC. Thus, all studies that were investigating the detection capabilities of CBCT [19-23] were focusing on primary liver cancer and to our knowledge no study on the visibility of liver metastases on CBCT was published. However, liver metastases are often hypovascular and thus their visibility on DSA is not as conspicuous as compared to HCC. Thus, it is important to investigate and optimize the capabilities of CBCT for the intraprocedural visualization of liver metastases so that CBCT can facilitate IAT of liver metastases as well. Therefore, the purpose of our study was to compare the visibility of liver metastases on dual-phase cone-beam CT (DP-CBCT) and DSA with reference to preinterventional CE-MRI of the liver.

\section{Materials and Methods}

\section{Study Cohort}

This single-center, retrospective study was compliant with the Health Insurance Portability and Accountability Act and was approved by the Institutional Review Board. Informed consent was waived. All patients referred to IAT were discussed at our multidisciplinary liver tumor board. Between January 2010 and October 2014, a total of 1488 IATs were performed in 962 patients with primary or secondary liver cancer at our institution. Inclusion criteria for IAT were as follows: Eastern Cooperative Oncology Group (ECOG) performance status $\leq 2$; Child-Pugh classification A or B; focal or multifocal hepatic malignancy; no severe ascites; albumin $>2.5 \mathrm{~g} / \mathrm{dl}$; alanine aminotransferase and aspartate aminotransferase $<5$ times the upper normal limit; total serum bilirubin $<3.0 \mathrm{mg} / \mathrm{dl}$; serum creatinine $<2.0 \mathrm{mg} / \mathrm{dl}$; platelet count $\geq 50,000 / \mathrm{mm}^{3}$; international normalized ratio $\leq 1.5$; and left ventricular ejection fraction $\geq 50 \%$.

The majority of the patients had primary liver cancer being either hepatocellular $(n=661)$ or cholangiocellular 
$(n=66)$ carcinoma. The most common liver metastases were from neuroendocrine cancer (NELM; $n=166$ ), followed by colorectal cancer (CRCLM; $n=53$ ) and sarcoma (SLM; $n=16$ ). All patients with liver metastases that were referred to our department had liver-dominant disease and had shown progression of the liver metastases during systemic therapy, thus intra-arterial procedures were performed as salvage therapies.

In 515 out of 1488 IAT procedures, a DP-CBCT was acquired to facilitate the optimal placement of the treatment catheter, 98 of these DP-CBCTs were acquired during an IAT of secondary liver cancer. To avoid statistical bias due to repeated measurements in patients who received more than one IAT procedure, only the first IAT of each patient with secondary liver cancer was included, resulting in 50 DP-CBCTs for further analysis.

21 patients with more than ten lesions were excluded due to limited capabilities of two-dimensional DSA to distinguish individual lesions in such patients. Another patient was excluded because he showed severe disease progression between baseline MRI and IAT.

On the basis of these criteria, the final study population included 28 patients, who were treated by conventional TACE (cTACE; $n=13$ ), radio-embolization (RE; $n=9$ ), and drug-eluting beads TACE (DEB-TACE; $n=6$ ), respectively. For cTACE procedures, a solution containing $100 \mathrm{mg}$ of cisplatin, $50 \mathrm{mg}$ of doxorubicin, and $10 \mathrm{mg}$ of mitomycin $\mathrm{C}$ in a 1:1 mixture with Lipiodol (Guerbet, France) was injected, followed by the administration of 100 - to 300- $\mu \mathrm{m}$-diameter microspheres (Embospheres, Merit Medical, USA). For RE, a shunt scan was performed using 5-6 mCi of 99mTC-labeled macroaggregated albumin at least 1 week prior to the infusion of Y90 microspheres (TheraSpheres ${ }^{\circledR}$, MDS Nordion, Ottawa, Canada). For DEB-TACE, LC Beads ( $2 \mathrm{~mL}$; BTG, Surrey, England) with a diameter of 100-300 mm were loaded with $100 \mathrm{mg}$ of doxorubicin hydrochloride $(25 \mathrm{mg} / \mathrm{mL})$ and mixed with an equal volume of nonionic contrast material. Up to $4 \mathrm{~mL}$ of drug-eluting beads was administered. Baseline characteristics are summarized in Table 1.

\section{MR Imaging Technique}

All study patients underwent baseline MRI within 2 months before IAT (median 20 days, range 0-61) using a 1.5-T MRI unit (Magnetom Avanto, Siemens Medical Solutions, Forchheim, Germany). A phased-array torso coil was used for signal reception. Our institutional liver protocol was performed including axial T2-weighted fast spinecho images, axial single-shot breath-hold gradient-echo diffusion-weighted echo-planar images, and axial breathhold unenhanced and contrast-enhanced $(0.1 \mathrm{mmol} / \mathrm{kg}$ intravenous gadodiamide [Omniscan; Amersham, Princeton,
Table 1 Baseline characteristics of the study cohort $(n=28)$

\begin{tabular}{|c|c|}
\hline Characteristic & Value $(\%)$ \\
\hline No. of patients & $28(100)$ \\
\hline \multicolumn{2}{|l|}{ Sex } \\
\hline Female & $12(42.9)$ \\
\hline Male & $16(57.1)$ \\
\hline \multicolumn{2}{|l|}{ Age* } \\
\hline All patients & $59 \pm 12$ years \\
\hline Female & $59 \pm 14$ years \\
\hline Male & $59 \pm 9$ years \\
\hline \multicolumn{2}{|c|}{ Eastern cooperative oncology group performance status } \\
\hline Grade 0 & $14(50.0)$ \\
\hline Grade 1 & $10(35.7)$ \\
\hline Grade 2 & $4(14.3)$ \\
\hline \multicolumn{2}{|c|}{ Origin of hepatic metastases } \\
\hline Neuroendocrine cancer & $15(53.6)$ \\
\hline Colorectal cancer & $10(35.7)$ \\
\hline Sarcoma & $3(10.7)$ \\
\hline \multicolumn{2}{|l|}{ Number of hepatic lesions } \\
\hline 1 & $3(10.7)$ \\
\hline $2-4$ & $11(39.3)$ \\
\hline $5-10$ & $14(50.0)$ \\
\hline \multicolumn{2}{|l|}{ Hepatic metastases location } \\
\hline Right lobe & $10(35.7)$ \\
\hline Left lobe & $1(3.6)$ \\
\hline Bilobar & $17(60.7)$ \\
\hline \multicolumn{2}{|l|}{ Extrahepatic metastases } \\
\hline Lymph nodes & $14(50.0)$ \\
\hline Lung & $7(25.0)$ \\
\hline Bones & 3 (10.7) \\
\hline
\end{tabular}

Except where indicated, data represents numbers of patients, and numbers in parentheses are percentages

* Data represented as mean \pm standard deviation

$\mathrm{NJ}$ ) T1-weighted three-dimensional (3D) fat-suppressed spoiled gradient-echo images in the arterial, portal venous, and delayed phases $(20,70$, and $180 \mathrm{~s}$ after intravenous contrast administration, respectively).

\section{Intraprocedural Imaging (DSA and C-Arm DP- CBCT)}

All IAT procedures were performed by a single interventional radiologist (JFG) with 19 years of experience in hepatic interventions, using our standard institutional protocol [24]. Briefly, access was gained in the femoral artery using the Seldinger technique. The celiac axis was then cannulated using a 5-F Simmons-1 catheter (Cordis, Miami Lakes, FL, USA) through which a 2.8 F Renegade HI-FLO microcatheter (Boston Scientific, Marlborough, MA, USA) was coaxially advanced. Several angiographic steps were 
performed to define the hepatic arterial anatomy, to determine portal venous patency and tumor enhancement. Injection rates were adapted to the estimated blood vessel diameter $(1-3 \mathrm{ml} / \mathrm{s})$.

All procedures were performed using an angiographic system (Allura Xper FD20, Philips Healthcare, Best, The Netherlands) equipped with the XperCT module, enabling $\mathrm{C}$-arm CBCT acquisition and volumetric image reconstruction (Feldkamp back projection) [25], and the DPCBCT prototype feature, allowing the acquisition of two sequential CBCT scans (in an early and a delayed arterial phase (EAP and DAP)) using only one contrast injection [26, 27]. Contrast injections (Oxilan $300 \mathrm{mg} \mathrm{I} / \mathrm{ml}$; Guerbet, France) were performed with a power injector (Medrad, Indianola, PA, USA). All patients underwent C-arm DPCBCT with the microcatheter placed into the hepatic artery branch that led to the tumor-feeding vessels and was in the same position as the last-acquired DSA, just prior to the delivery of the chemo-embolic (for TACE) or diagnostic (for shunt scan performed prior to RE) agents. In particular, the position of the microcatheter tip was lobar and segmental in $13(46.4 \%)$ and $15(53.6 \%)$ patients, respectively. The area of interest was positioned in the system isocenter prior to the DP-CBCT scan. The acquisition parameters were set to $120 \mathrm{kVp}$ tube voltage and $50-325 \mathrm{~mA}$ tube current, the latter being modulated automatically during the acquisition. The two scans were triggered at 3 and $28 \mathrm{~s}$ after a single injection of $20 \mathrm{ml}$ of undiluted contrast agent with a flow rate of $2 \mathrm{ml} / \mathrm{s}$. The patients were instructed to be at endexpiration apnea during each of the CBCT scans with free breathing between the early and the delayed arterial phase scans. Oxygen was administered to patients during the procedure to minimize the discomfort of breath holding. With the motorized C-arm covering a $240^{\circ}$ clockwise arc at a rotation speed of up to $55^{\circ} / \mathrm{s}, 312$ projection images ( 60 frames/s) were acquired in $5.2 \mathrm{~s}$. On completion of the acquisition, the two-dimensional projections were automatically transferred to the reconstruction computer, where they were reconstructed into 3D volumetric images with an isotropic resolution of $0.65 \mathrm{~mm}^{3}$, a field of view (FOV) of $250^{2} \times 194 \mathrm{~mm}$, and a matrix size of $384^{2} \times 296$.

\section{Image Analysis}

Image analysis was performed using a free viewer software (Osirix, Pixmeo, Bernex, Switzerland) by two interventional radiologists, both with 4 years of experience (RES and RD), who did not participate in the IAT procedures. The observers were allowed to alter the window/level and zoom levels of the images to optimize perception. Streak artifacts caused by breathing, the intra-arterial catheter, and other medical devices (e.g., intravenous catheters) were assessed on DP-CBCT images using a three-point scale (none, localized, extensive). Extensive artifacts were considered to affect the diagnostic quality of the CBCT scan, whereas the presence of localized artifacts was deemed acceptable for diagnosis.

151 hepatic metastases were identified on preinterventional CE-MRI. 4 lesions were outside the FOV of the $\mathrm{CBCT}$ acquisitions and were excluded from the analysis. In addition, because the CBCTs were not acquired from the proper hepatic artery, but rather more selectively from within the liver vasculature, only lobar or segmental contrast attenuation of the liver parenchyma was seen. Thus, lesions that were entirely situated in liver segments, not opacified by the contrast medium injection during the CBCT acquisition were excluded. Of note, lesions that had a dual supply from both the left and right hepatic arteries were not excluded if the injected contrast medium reached the tumor from one of the feeding arteries. Following this approach, 41 lesions were excluded, leaving a total of 106 lesions for final analysis.

Each lesion was examined on the arterial and the portal venous phase of the preinterventional MRI and the lesion diameters were measured on the phase offering the best visualization of the lesion's rim. Using this MRI phase as a side-by-side reference, the visibility of each lesion on DSA, EAP-, and DAP-CBCT was ranked according to the following scoring system: (1) optimal = the lesion was clearly detectable such as that in CE-MRI; (2) suboptimal = complete extent of the lesion was not visible; and (3) nondiagnostic $=$ the lesion could not be detected at all.

\section{Statistical Analysis}

All statistical computations were performed in SPSS Statistics 22 (IBM Corp., Armonk, NY). A $p$ value less than 0.05 was considered statistically significant. Descriptive statistics were used to summarize the data. The distribution of all scale variables was assessed with the Shapiro-Wilk test. Scale variables with normal distribution were expressed with mean and standard deviation. For scale variables with nonGaussian distribution, median and range were reported. For ordinal variables, frequencies and percentage were used. Statistical significance was assessed with Friedman's two-way ANOVA. In addition, binary testing of detected vs. not detected was performed using Cochran's Q test after combining the categories for partial and complete depiction into one group.

\section{Results}

On all CBCT images, localized streak artifacts were caused by contrast-filled catheters and contrast-enhanced arteries within the liver. The majority of EAP- and DAP-CBCT 
images showed no breathing artifacts (71 and $57 \%$, respectively), localized breathing artifacts were present in the remaining cases. In two patients (7\%), localized streak artifacts caused by the central venous catheter in the right atrium of the heart were observed. There were no extensive artifacts due to breathing, contrast-filled catheters, or other implants. Thus, all CBCT images were of diagnostic quality.

The median size of all metastatic liver lesions was $20 \mathrm{~mm}$ (range, 7-154 mm), with NELM lesions being slightly bigger (median $26 \mathrm{~mm}$, range $10-116 \mathrm{~mm}$ ) than SLM lesions (median $19 \mathrm{~mm}$, range $8-100 \mathrm{~mm}$ ) and CRCLM lesions (median $16 \mathrm{~mm}$, range $7-154 \mathrm{~mm}$ ). Out of the 106 lesions, only 65 (61.3\%) could be identified on DSA images, whereas EAP- and DAP-CBCT images depicted $94(88.7 \%)$ and $106(100.0 \%)$ lesions, respectively. Combining all metastatic liver lesions together, Cochran's Q test showed that both EAP- and DAP-CBCT yielded significantly superior detectability compared to DSA $(p<0.01)$. Looking at each tumor entity separately, EAP-CBCT had only significant benefit for the detection of CRCLM and SLM, but not NELM, whereas DAP-CBCT was significantly better than DSA in detecting all the three metastases types (Table 2). However, DAP-CBCT had no significant advantage over EAP-CBCT for the detection of all lesions $(p=0.085)$, CRCLM $(p=0.689)$, NELM $(p=0.091)$, and SLM $(p=1.0)$.

More specifically, a complete depiction was achieved by DSA, EAP-, and DAP-CBCT in 38 (35.8\%), 43 (40.6\%), and $103(97.2 \%)$ liver metastases, respectively. Partial depiction was achieved on DSA, EAP-, and DAP-CBCT images for $27(25.5 \%), 51(48.1 \%)$, and $3(2.8 \%)$ liver metastases, respectively. Friedman's Two-Way ANOVA showed a significant advantage of DAP-CBCT over EAP-
CBCT $(p<0.001)$ and DSA $(p<0.001)$, respectively, whereas the difference between EAP-CBCT and DSA was not significant $(p=0.298)$. Looking at each tumor entity separately, EAP-CBCT significantly outperformed DSA only for the complete depiction of CRCLM, but not of NELM and SLM (Table 2). DAP-CBCT on the other hand, was not only significantly better than DSA for the complete depiction of all the three types of metastatic liver lesion, but also better than EAP-CBCT for the complete delineation of CRCLM $(p=0.008)$, NELM $(p<0.001)$, and $\operatorname{SLM}(p=0.031)$.

All 41 lesions missed by DSA were detected by DAPCBCT, $39(95 \%)$ and $2(5 \%)$ being completely and partially depicted, respectively (Fig. 1). Out of these 41 lesions, $15(36.6 \%)$ and $18(43.9 \%)$ were completely and partially depicted on EAP-CBCT, respectively (Fig. 2). DAP-CBCT depicted 12 more lesions than EAP-CBCT, whereas EAP-CBCT did not show any additional lesions compared to DAP-CBCT. All four lesions, that were missed on EAP-CBCT, but visible on DSA, were completely depicted on DAP-CBCT (Fig. 3).

\section{Discussion}

The main finding of our study was that DAP-CBCT substantially improved the detectability of all three entities of metastatic liver lesions during IAT procedures. Using conventional DSA alone, almost $40 \%$ of the liver metastases could not be identified. Whereas EAP-CBCT missed only approximately $10 \%$ of the liver metastases and DAPCBCT depicted all of them. Most likely, without the additional information provided by the CBCT scans, a less selective treatment (e.g., lobar application) would have
Table 2 Detectability scores cross table of liver metastases on digital subtraction angiography (DSA), early arterial and delayed arterial phase (EAP- and DAP-) CBCT

\begin{tabular}{|c|c|c|c|c|c|c|c|c|c|c|c|c|}
\hline \multirow[t]{2}{*}{ Cancer type } & & & \multicolumn{5}{|c|}{ ЕАР-СВCТ } & \multicolumn{5}{|c|}{ DAP-CBCT } \\
\hline & & & 1 & 2 & 3 & Q & ANOVA & 1 & 2 & 3 & Q & ANOVA \\
\hline \multirow[t]{3}{*}{ Colorectal cancer } & DSA & 1 & 3 & 3 & 1 & $<0.001$ & 0.008 & 7 & 0 & 0 & $<0.001$ & 0.008 \\
\hline & & 2 & 5 & 6 & 1 & & & 11 & 1 & 0 & & \\
\hline & & 3 & 12 & 9 & 3 & & & 23 & 1 & 0 & & \\
\hline \multirow{3}{*}{$\begin{array}{l}\text { Neuroendocrine } \\
\text { cancer }\end{array}$} & DSA & 1 & 15 & 13 & 0 & 1.0 & 0.592 & 28 & 0 & 0 & 0.007 & 0.008 \\
\hline & & 2 & 3 & 8 & 1 & & & 12 & 0 & 0 & & \\
\hline & & 3 & 1 & 2 & 4 & & & 6 & 1 & 0 & & \\
\hline \multirow[t]{3}{*}{ Sarcoma } & DSA & 1 & 2 & 0 & 1 & 0.009 & 0.399 & 3 & 0 & 0 & 0.001 & $<0.001$ \\
\hline & & 2 & 0 & 3 & 0 & & & 3 & 0 & 0 & & \\
\hline & & 3 & 2 & 7 & 1 & & & 10 & 0 & 0 & & \\
\hline
\end{tabular}

Detectability scores: 1 = complete depiction; 2 = partial depiction; $3=$ no depiction

Q corresponds to Cochran's Q test, performed after binary conversion of the scores $(1+2=$ detected; $3=$ not detected)

ANOVA corresponds to Friedman's two-way ANOVA 


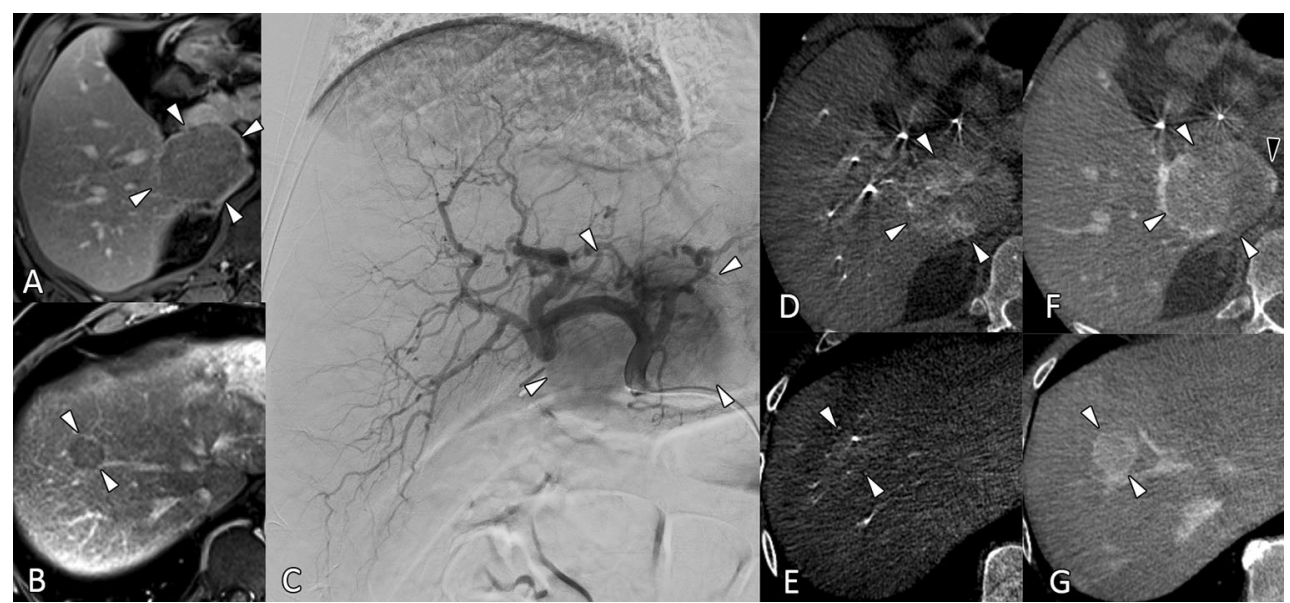

Fig. 1 54-year-old man with a history of neuroendocrine cancer of the small bowel with liver metastases, treated using conventional TACE. Contrast-enhanced T1-weighted gradient-echo sequence in the portal venous phase shows a large, rim-enhancing lesion in the caudate lobe (A, arrowheads) and a smaller lesion of similar pattern in segment 8 ( $\mathbf{B}$, arrowheads). On DSA images acquired with the microcatheter tip in the proper hepatic artery, only the large lesion could be identified (C, arrowheads). On early arterial phase CBCT images, only the lateral parts of the large lesion are depicted (D, arrowheads), the smaller lesion is only silhouetted against the surrounding parenchyma (E, arrowheads). On delayed arterial phase CBCT images, the large lesion is well depicted (F) to include both the lateral parts (white arrowheads) as well as the medial rim (black arrowhead). Of note, the small lesion is completely depicted (G, arrowheads)
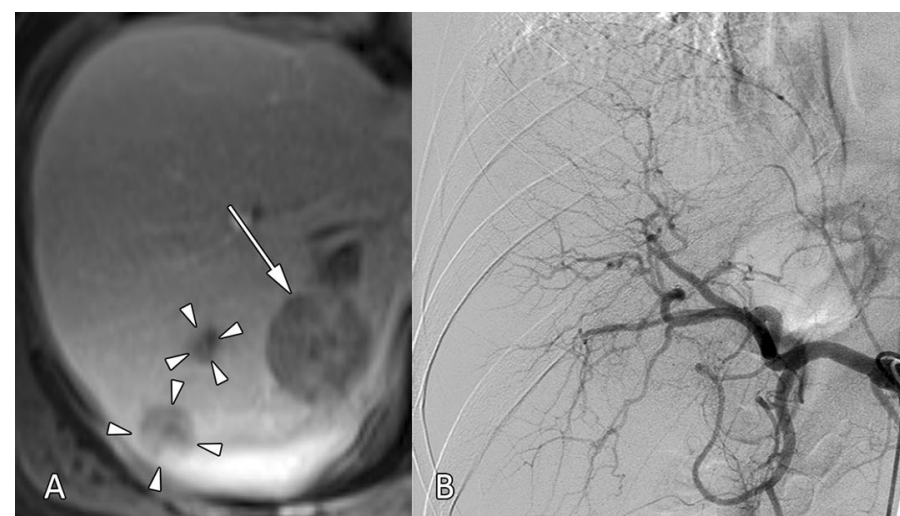

Fig. 2 43-year-old man with a history of retroperitoneal sarcoma with liver metastases, treated using conventional TACE. A Contrastenhanced T1-weighted gradient-echo sequence in the portal venous phase shows three lesions in segment 7, one larger (arrow) and two smaller tumors (arrowheads). B On the celiac arteriogram, none of

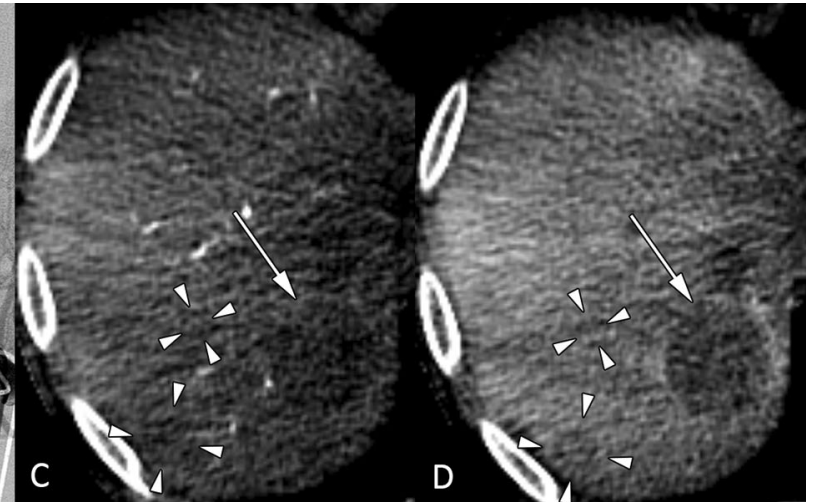

the lesions is visible. $\mathbf{C}$ On early arterial phase $\mathrm{CBCT}$, the large lesion is well depicted (arrow), but the two smaller lesions are difficult to distinguish (arrowheads). D On delayed arterial phase CBCT, all three lesions are well depicted (arrow and arrowheads)

intrahepatic cholangiocarcinoma (ICC) lesions on DPCBCT, using CE-MRI as Ref. [23]. This study showed that due to delayed enhancement pattern of ICC lesions, as seen on DSA and MRI [29, 30], DAP-CBCT was substantially better in depicting ICC lesions than EAP-CBCT and DSA. Similar to ICC, most metastatic liver lesions are rather hypovascular and show delayed enhancement pattern which is often limited to a rim around a necrotic core, whereas NELM are often hypervascular lesions $[10,11]$. Thus, less than $50 \%$ of CRCLM and SLM lesions, but $85 \%$ of NELM could be identified on DSA in our study. On CBCT, most lesions showed minimal to no 


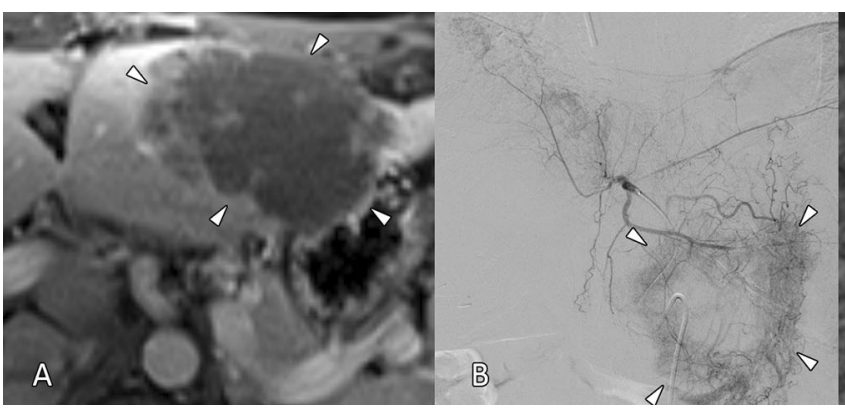

Fig. 3 44-year-old woman with a history of colorectal cancer with liver metastases, treated using radio-embolization with Yttrium-90. A Contrast-enhanced T1-weighted gradient-echo sequence in the portal venous phase shows a large, mainly necrotic lesion with rim enhancement in segment 3. B On the DSA images acquired with the

enhancement on EAP-CBCT, with the lesion rim and the surrounding liver parenchyma enhancing on DAP-CBCT, demarcating the necrotic core of these lesions.

Although the detection rate for NELM was already quite high on DSA, DAP-CBCT reached a significantly higher detection rate for all the three entities of metastatic liver lesions, whereas EAP-CBCT outperformed DSA only for CRCLM and SLM, but not for NELM lesions.

Recent publications have shown that intraprocedural CBCT does not only facilitate the positioning of the delivery catheter for optimal targeting of the tumor [31], but also provides intraprocedural feedback on the technical success of the IAT procedure by means of three-dimensional quantification of contrast enhancement/deposition $[32,33]$. For that purpose, a partial depiction of intrahepatic lesions is not sufficient, only lesions with a complete depiction can be evaluated using this new approach. In our study, EAP-CBCT delineated only $40 \%$ of the lesions completely, whereas DAP-CBCT succeeded in $97 \%$. This underlines the importance of an optimized CBCT acquisition protocol, based on tumor enhancement patterns.

Additional radiation exposure is often considered a severe drawback of CBCT. However, a recently published trial that investigated the radiation exposure during TACE showed that CBCT accounts for only approximately $10 \%$ of the radiation exposure during the entire procedure using standard equipment [34]. In particular, a single-phase CBCT corresponds to approximately $150 \mathrm{~s}$ of digital fluoroscopy or $4 \mathrm{~s}$ of DSA. A CBCT run with the catheter tip in the proper hepatic artery could be used as the source of a three-dimensional overlay for intraprocedual guidance and could in theory replace all intrahepatic DSA runs, thereby reducing both radiation exposure and contrast volume. However, this needs to be confirmed in a prospective trial.

The present study has some limitations: First, being the small sample size with metastases of different origins. However, the number of patients with secondary liver

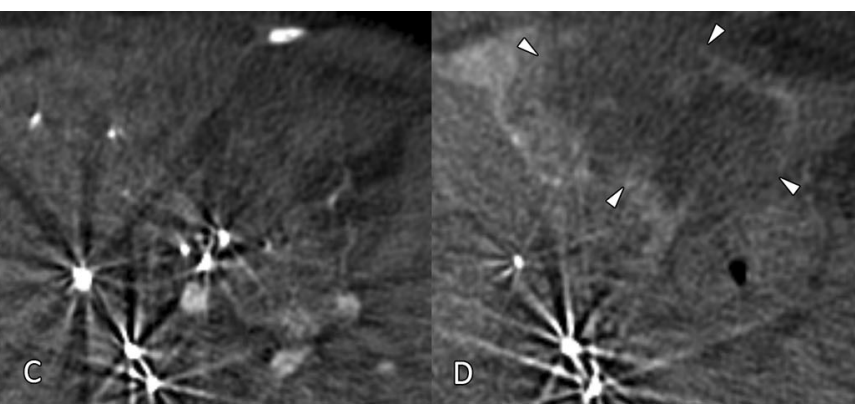

microcatheter tip in the left hepatic artery, the lesion is well depicted. C However, on early arterial phase CBCT images, the lesion is not visible. D On delayed arterial phase CBCT images, the entire extent of the lesion is well depicted (arrowheads)

cancer being treated by means of IAT is rather small compared to the patient population with hepatocellular carcinoma. In addition, $44 \%$ of the patients had to be excluded due to extensive disease, limiting the diagnostic capabilities of DSA. Although a less selective approach for IAT might be indicated in these patients, CBCT should still be performed to verify if known lesions have progressed or new liver lesions have emerged because this could modify the treatment plan to either a less selective drug delivery or to have additional selective catheter positions for drug delivery. Second, in the absence of a control group of patients with liver metastases treated using IAT without $\mathrm{CBCT}$, the impact of CBCT on radiological response or overall outcome could not be evaluated within this retrospective study. However, the evaluation of the diagnostic accuracy of a new intraprocedural imaging modality is a prerequisite before prospective trials are conducted to assess the clinical impact. Third, the CBCTs were not acquired from the proper hepatic artery, but rather more selectively from within the liver vasculature, thus only lobar or segmental contrast attenuation of the liver parenchyma was seen and lesions in other segments as depicted by the CE-MRI had to be excluded. This also did not allow for investigation of the intraprocedural guidance capabilities of CBCT. However, the position of the catheter was selected based on the tumor burden as seen on the preinterventional CE-MRI. Fourth, some hepatic lesions had to be excluded because they were outside the FOV of the CBCT scan. This has been a common problem of CBCT until recently, with up to $12 \%$ of lesions being outside the FOV in the literature [23, 28]. However, a solution to this problem was recently demonstrated by changing the CBCT rotation trajectory while still maintaining the same number of projection images and rotational sweep angle [35]. Fifth, for each patient, only one DP-CBCT was acquired prior to the delivery of embolic agents, no DP-CBCT was acquired after the delivery. Thus, 
quantitative intraprocedural response assessment as described previously [32, 33] could not be performed.

Despite these limitations, our results demonstrated that the addition of a second CBCT phase significantly improved identification of metastatic liver lesions. Although EAPCBCT did not show any lesions missed by DAP-CBCT, the former is still necessary to visualize the feeding arteries in our current protocol [36]. However, an optimized DAPCBCT protocol is currently underdevelopment, that uses a prolonged contrast injection to facilitate the visualization of both the feeding arteries and the tumor parenchyma [37].

In conclusion, DAP-CBCT substantially improved the visibility of liver metastases during IAT. Future studies need to investigate whether this improved visibility facilitates a more selective treatment, resulting in a better radiological response and better overall outcome. In addition, the capabilities of CBCT for intraprocedural guidance should be evaluated, thereby having the potential to replace intrahepatic DSA runs in order to reduce radiation exposure and allow for the assessment of treatment success during IAT of liver metastases.

Acknowledgments Open access funding provided by Medical University of Vienna. Support for this work was provided by the Max Kade Foundation, Inc., NY, USA, NIH/NCI R01 CA160771, P30 CA006973, Philips Research North America, Cambridge, MA, USA. Ruediger E. Schernthaner: Grant Support: Max Kade Foundation, Inc., NY, USA. Alessandro Radaelli and Imramsjah Martijn van der Bom: employee: Philips Healthcare, Best, The Netherlands. MingDe Lin: Grant Support: NIH; employee: Philips Research North America, Cambridge, MA, USA. Jean Francois Geschwind: Consultant: Nordion, Biocompatibles/BTG, Bayer HealthCare; Grant Support: NIH, Philips Medical, DOB, Biocompatibles/BTG, Bayer HealthCare, Nordion, Context Vision, SIR, RSNA, Guerbet; Founder and CEO PreScience Labs, LLC.

\section{Compliance with Ethical Standards}

Ethical Approval All procedures performed in studies involving human participants were in accordance with the ethical standards of the institutional and/or national research committee and with the 1964 Helsinki declaration and its later amendments or comparable ethical standards.

Open Access This article is distributed under the terms of the Creative Commons Attribution 4.0 International License (http:// creativecommons.org/licenses/by/4.0/), which permits unrestricted use, distribution, and reproduction in any medium, provided you give appropriate credit to the original author(s) and the source, provide a link to the Creative Commons license, and indicate if changes were made.

\section{References}

1. Jemal A, Siegel R, Xu J, Ward E. Cancer statistics, 2010. CA Cancer J Clin. 2010;60(5):277-300.

2. Imam $\mathrm{K}$, Bluemke DA. MR imaging in the evaluation of hepatic metastases. Magn Reson Imaging Clin N Am. 2000;8(4):741-56.
3. Haddad AJ, Bani Hani M, Pawlik TM, Cunningham SC. Colorectal liver metastases. Int J Surg Oncol. 2011;2011:285840.

4. Krikelis D, Judson I. Role of chemotherapy in the management of soft tissue sarcomas. Expert Rev Anticancer Ther. 2010;10(2): 249-60.

5. Hong K, McBride JD, Georgiades CS, Reyes DK, Herman JM, Kamel IR, et al. Salvage therapy for liver-dominant colorectal metastatic adenocarcinoma: comparison between transcatheter arterial chemoembolization versus yttrium-90 radioembolization. J Vasc Interv Radiol. 2009;20(3):360-7.

6. Gruttadauria S, Zacharias AJ, Jayakrishnan TT, Rajeev R, Rilling WS, Thomas JP, et al. Comparative effectiveness of hepatic artery based therapies for unresectable colorectal liver metastases: a meta-analysis. PLoS One. 2015;10(10):e0139940.

7. Kennedy A, Bester L, Salem R, Sharma RA, Parks RW, Ruszniewski $\mathrm{P}$, et al. Role of hepatic intra-arterial therapies in metastatic neuroendocrine tumours (NET): guidelines from the NETliver-metastases consensus conference. HPB. 2015;17(1):29-37.

8. de Baere T, Arai Y, Lencioni R, Geschwind JF, Rilling W, Salem $\mathrm{R}$, et al. Treatment of liver tumors with lipiodol TACE: technical recommendations from experts opinion. Cardiovasc Interv Radiol. 2016;39(3):334-43.

9. Rajan DK, Soulen MC, Clark TW, Baum RA, Haskal ZJ, Shlansky-Goldberg RD, et al. Sarcomas metastatic to the liver: response and survival after cisplatin, doxorubicin, mitomycin-C, ethiodol, and polyvinyl alcohol chemoembolization. J Vasc Interv Radiol. 2001;12(2):187-93.

10. Elsayes KM, Narra VR, Yin Y, Mukundan G, Lammle M, Brown JJ. Focal hepatic lesions: diagnostic value of enhancement pattern approach with contrast-enhanced 3D gradient-echo MR imaging. Radiographics. 2005;25(5):1299-320.

11. Namasivayam S, Martin DR, Saini S. Imaging of liver metastases: mRI. Cancer Imaging. 2007;7:2-9.

12. Wallace MJ, Murthy R, Kamat PP, Moore T, Rao SH, Ensor J, et al. Impact of $\mathrm{C}$-arm CT on hepatic arterial interventions for hepatic malignancies. J Vasc Interv Radiol. 2007;18(12):1500-7.

13. Tognolini A, Louie JD, Hwang GL, Hofmann LV, Sze DY, Kothary N. Utility of C-arm CT in patients with hepatocellular carcinoma undergoing transhepatic arterial chemoembolization. J Vasc Interv Radiol. 2010;21(3):339-47.

14. Hirota S, Nakao N, Yamamoto S, Kobayashi K, Maeda H, Ishikura $\mathrm{R}$, et al. Cone-beam CT with flat-panel-detector digital angiography system: early experience in abdominal interventional procedures. Cardiovasc Interv Radiol. 2006;29(6):1034-8.

15. Wallace MJ, Kuo MD, Glaiberman C, Binkert CA, Orth RC, Soulez G, et al. Three-dimensional C-arm cone-beam CT: applications in the interventional suite. $\mathrm{J}$ Vasc Interv Radiol. 2008;19(6):799-813.

16. Kakeda S, Korogi Y, Ohnari N, Moriya J, Oda N, Nishino K, et al. Usefulness of cone-beam volume CT with flat panel detectors in conjunction with catheter angiography for transcatheter arterial embolization. J Vasc Interv Radiol. 2007; 18(12):1508-16.

17. Meyer BC, Frericks BB, Voges M, Borchert M, Martus P, Justiz $\mathrm{J}$, et al. Visualization of hypervascular liver lesions during TACE: comparison of angiographic C-arm CT and MDCT. AJR Am J Roentgenol. 2008;190(4):W263-9.

18. Wallace MJ. C-arm computed tomography for guiding hepatic vascular interventions. Techn Vasc Interv Radiol. 2007;10(1): $79-86$.

19. Miyayama S, Yamashiro M, Okuda M, Yoshie Y, Sugimori N, Igarashi $\mathrm{S}$, et al. Usefulness of cone-beam computed tomography during ultraselective transcatheter arterial chemoembolization for small hepatocellular carcinomas that cannot be demonstrated on angiography. Cardiovasc Interv Radiol. 2009;32(2):255-64. 
20. Miyayama S, Yamashiro M, Hattori Y, Orito N, Matsui K, Tsuji $\mathrm{K}$, et al. Efficacy of cone-beam computed tomography during transcatheter arterial chemoembolization for hepatocellular carcinoma. Jpn J Radiol. 2011;29(6):371-7.

21. Miyayama S, Yamashiro M, Okuda M, Yoshie Y, Nakashima Y, Ikeno $\mathrm{H}$, et al. Detection of corona enhancement of hypervascular hepatocellular carcinoma by $\mathrm{C}$-arm dual-phase cone-beam CT during hepatic arteriography. Cardiovasc Interv Radiol. 2011;34(1): $81-6$.

22. Loffroy R, Lin M, Rao P, Bhagat N, Noordhoek N, Radaelli A, et al. Comparing the detectability of hepatocellular carcinoma by $\mathrm{C}$-arm dual-phase cone-beam computed tomography during hepatic arteriography with conventional contrast-enhanced magnetic resonance imaging. Cardiovasc Interv Radiol. 2012;35(1):97-104.

23. Schernthaner RE, Lin M, Duran R, Chapiro J, Wang Z, Geschwind JF. Delayed-phase cone-beam CT improves detectability of intrahepatic Cholangiocarcinoma during conventional transarterial chemoembolization. Cardiovasc Interv Radiol. 2015;38(4): 929-36.

24. Liapi E, Geschwind JF. Transcatheter arterial chemoembolization for liver cancer: is it time to distinguish conventional from drugeluting chemoembolization? Cardiovasc Interv Radiol. 2011;34(1): 37-49.

25. Feldkamp L, Davis L, Kress J. Practical cone-beam algorithms. J Opt Soc Am A. 1984;6:612-9.

26. Lin M, Loffroy R, Noordhoek N, Taguchi K, Radaelli A, Blijd J, et al. Evaluating tumors in transcatheter arterial chemoembolization (TACE) using dual-phase cone-beam CT. Minim Invasive Ther Allied Technol. 2011;20(5):276-81.

27. Tacher V, Radaelli A, Lin M, Geschwind JF. How i do it: conebeam CT during transarterial chemoembolization for liver cancer. Radiology. 2015;274(2):320-34.

28. Miyayama S, Matsui O, Yamashiro M, Ryu Y, Takata H, Takeda T, et al. Detection of hepatocellular carcinoma by CT during arterial portography using a cone-beam CT technology: comparison with conventional CTAP. Abdom Imaging. 2009;34(4):502-6.

29. Sainani NI, Catalano OA, Holalkere NS, Zhu AX, Hahn PF, Sahani DV. Cholangiocarcinoma: current and novel imaging techniques. Radiographics. 2008;28(5):1263-87.
30. Jeong HT, Kim MJ, Chung YE, Choi JY, Park YN, Kim KW. Gadoxetate disodium-enhanced MRI of mass-forming intrahepatic cholangiocarcinomas: imaging-histologic correlation. AJR Am J Roentgenol. 2013;201(4):W603-11.

31. Miyayama S, Yamashiro M, Hashimoto M, Hashimoto N, Ikuno $\mathrm{M}$, Okumura $\mathrm{K}$, et al. Identification of small hepatocellular carcinoma and tumor-feeding branches with cone-beam CT guidance technology during transcatheter arterial chemoembolization. J Vasc Interv Radiol. 2013;24(4):501-8.

32. Loffroy R, Lin M, Yenokyan G, Rao PP, Bhagat N, Noordhoek $\mathrm{N}$, et al. Intraprocedural $\mathrm{C}$-arm dual-phase cone-beam CT: can it be used to predict short-term response to TACE with drug-eluting beads in patients with hepatocellular carcinoma? Radiology. 2013;266(2):636-48.

33. Wang Z, Chen R, Duran R, Zhao Y, Yenokyan G, Chapiro J, et al. Intraprocedural 3D quantification of lipiodol deposition on cone-beam CT predicts tumor response after transarterial chemoembolization in patients with hepatocellular carcinoma. Cardiovasc Interv Radiol. 2015;38(6):1548-56.

34. Schernthaner RE, Duran R, Chapiro J, Wang Z, Geschwind JF, Lin M. A new angiographic imaging platform reduces radiation exposure for patients with liver cancer treated with transarterial chemoembolization. Eur Radiol. 2015;25(11):3255-62.

35. Schernthaner RE, Chapiro J, Sahu S, Withagen P, Duran R, Sohn $\mathrm{JH}$, et al. Feasibility of a modified cone-beam CT rotation trajectory to improve liver periphery visualization during transarterial chemoembolization. Radiology. 2015;277(3):833-41.

36. Tacher V, Lin M, Bhagat N, Abi Jaoudeh N, Radaelli A, Noordhoek N, et al. Dual-phase cone-beam computed tomography to see, reach, and treat hepatocellular carcinoma during drugeluting beads transarterial chemo-embolization. J Vis Exp. 2013;82:50795.

37. van den Hoven AF, Prince JF, de Keizer B, Vonken EJ, Bruijnen $\mathrm{RC}$, Verkooijen HM, et al. Use of C-arm cone beam CT during hepatic radioembolization: protocol optimization for extrahepatic shunting and parenchymal enhancement. Cardiovasc Interv Radiol. 2016;39(1):64-73. 